\title{
Polar pollutants entry into the water cycle by municipal wastewater: a European perspective
}

Thorsten Reemtsma $^{1 *}$, Stefan Weiss ${ }^{1}$, Jutta Mueller ${ }^{2}$, Mira Petrovic $^{3}$, Susana González ${ }^{3}$, Francesc Ventura ${ }^{4}$, Thomas P. Knepper ${ }^{2 *}$

1) Technical University of Berlin, Department of Water Quality Control, Sekr KF 4, Strasse des 17 Juni 135, 10623 Berlin, Germany

2) Europa University for Applied Sciences Fresenius, Limburger Straße 2, 65510 Idstein, Germany.

3) Department of Environmental Chemistry, IIQAB-CSIC, Jordi Girona 18-26, 08034 Barcelona, Spain.

4) Aigües de Barcelona. Avinguda Diagonal 211. E-08018-Barcelona, Spain.

Corresponding authors:

Thorsten Reemtsma; E-mail: thorsten.reemtsma@tu-berlin.de

Thomas P. Knepper; E-mail: knepper@fh-fresenius.de

\begin{abstract}
This supporting information provides additional data on the target analytes and the analytical methods used in the monitoring campaign (Tab. S1) and on the wastewater treatment plants investigated in this study (Tab. S2). Additionally some exemplary calculations of Water Cycle Spreading Indeces (WCSI) are provided to illustrate the dependency of this parameter on influent and effluent concentrations of a compound and on its extent of removal in wastewater treatment.
\end{abstract}


Table S1: Analytes included in this study, their methods of analysis and limits of quantification (LOQ)

\begin{tabular}{|c|c|c|c|c|c|c|}
\hline Analytes & Acronym & CAS-No. & Method & $\begin{array}{l}\text { Ref. } \\
\text { No. }\end{array}$ & $\begin{array}{l}\text { LOQ } \\
{[\mathrm{Fg} / \mathrm{L}]}\end{array}$ & study $^{2)}$ \\
\hline sulfophenylcarboxylates ${ }^{1)}$ & SPC & & LC-MS & {$[\mathrm{S} 1]$} & 0.3 & $\mathrm{M}, \mathrm{W}$ \\
\hline ethylendiamine tetraacetate & EDTA & $60-004$ & IC-MS & {$[\mathrm{S} 2]$} & 1.0 & $\mathrm{M}, \mathrm{W}$ \\
\hline nonylphenol ethoxylates & NP3-15EO & $9016-45-9$ & LC-MS & {$[\mathrm{S} 3]$} & 0.005 & $\mathrm{M}, \mathrm{W}$ \\
\hline nonylphenol diethoxylate & NP2EO & 20427-84-3 & LC-MS & {$[\mathrm{S} 3]$} & 0.05 & $\mathrm{M}, \mathrm{W}$ \\
\hline nonylphenol monoethoxylate & NP1EO & $104-35-8$ & LC-MS & {$[\mathrm{S} 3]$} & 0.05 & $\mathrm{M}, \mathrm{W}$ \\
\hline $\begin{array}{l}\text { nonylphenolmonoethoxy } \\
\text { carboxylate }\end{array}$ & NP1EC & $3115-49-9$ & LC-MS & {$[\mathrm{S} 3]$} & 0.1 & $\mathrm{M}, \mathrm{W}$ \\
\hline $\begin{array}{l}\text { nonylphenoldiethoxy } \\
\text { carboxylate }\end{array}$ & NP2EC & & LC-MS & [S3] & 0.1 & $\mathrm{M}, \mathrm{W}$ \\
\hline 4-nonylphenol & NP & $104-40-5$ & LC-MS & [S3] & 0.05 & $\mathrm{M}, \mathrm{W}$ \\
\hline $\begin{array}{l}\text { octylphenol } \\
\text { monoethoxycarboxylate }\end{array}$ & OP1EC & & LC-MS & [S3] & 0.1 & W \\
\hline $\begin{array}{l}\text { octylphenol } \\
\text { diethoxycarboxylate }\end{array}$ & OP2EC & & LC-MS & {$[\mathrm{S} 3]$} & 0.1 & $\mathrm{~W}$ \\
\hline 4-tert.octylphenol & $\mathrm{OP}$ & $140-66-9$ & LC-MS & {$[\mathrm{S} 3]$} & 0.05 & $\mathrm{~W}$ \\
\hline benzothiazole-2-sulfonate & BTSA & $941-57-1$ & LC-MS/MS & [S4] & 0.1 & $\mathrm{M}, \mathrm{W}$ \\
\hline benzothiazole & BT & $95-16-9$ & LC-MS/MS & [S4] & 0.1 & $\mathrm{M}, \mathrm{W}$ \\
\hline
\end{tabular}




\begin{tabular}{|c|c|c|c|c|c|c|}
\hline 2-hydroxybenzothiazole & OHBT & 934-34-9 & LC-MS/MS & {$[\mathrm{S} 4]$} & 0.2 & $\mathrm{M}, \mathrm{W}$ \\
\hline 2-mercapto benzothiazole & MBT & $149-30-4$ & LC-MS/MS & {$[\mathrm{S} 4]$} & 0.05 & $\mathrm{M}, \mathrm{W}$ \\
\hline 2-methylthio benzothiazole & MTBT & $612-22-5$ & LC-MS/MS & {$[\mathrm{S} 4]$} & 0.04 & $\mathrm{M}, \mathrm{W}$ \\
\hline 2-amino benzothiazole & ABT & $136-95-8$ & LC-MS/MS & {$[\mathrm{S} 4]$} & 0.03 & M, W \\
\hline benzotriazole & BTri & $95-14-7$ & LC-MS/MS & {$[\mathrm{S} 5]$} & 0.01 & $\mathrm{M}, \mathrm{W}$ \\
\hline 4-/5-tolyltriazole & TTri & $136-85-6$ & LC-MS/MS & {$[\mathrm{S} 5]$} & 0.01 & $\mathrm{M}, \mathrm{W}$ \\
\hline dimethylbenzotriazole & XTri & $4184-79-6$ & LC-MS/MS & {$[\mathrm{S} 5]$} & 0.01 & W \\
\hline naphthalene-2-sulfonate & 1-NSA & $120-18-3$ & LC-MS/MS & {$[\mathrm{S} 6]$} & 0.01 & $\mathrm{M}, \mathrm{W}$ \\
\hline naphthalene-1-sulfonate & 2-NSA & $85-47-2$ & LC-MS/MS & {$[\mathrm{S} 6]$} & 0.01 & $\mathrm{M}, \mathrm{W}$ \\
\hline naphthalene-1,6-disulfonate & 16-NDSA & $525-37-1$ & LC-MS/MS & {$[\mathrm{S} 6]$} & 0.02 & $\mathrm{M}, \mathrm{W}$ \\
\hline naphthalene-1,7-disulfonate & 17-NDSA & & LC-MS/MS & [S6] & 0.02 & $\mathrm{M}, \mathrm{W}$ \\
\hline naphthalene-2,6-disulfonate & 26-NDSA & $581-75-9$ & LC-MS/MS & {$[\mathrm{S} 6]$} & 0.02 & $\mathrm{M}, \mathrm{W}$ \\
\hline naphthalene-2,7-disulfonate & 27-NDSA & $92-41-1$ & LC-MS/MS & {$[\mathrm{S} 6]$} & 0.02 & $\mathrm{M}, \mathrm{W}$ \\
\hline naphthalene-1,5-disulfonate & 15-NDSA & $81-04-9$ & LC-MS/MS & {$[\mathrm{S} 6]$} & 0.02 & $\mathrm{M}, \mathrm{W}$ \\
\hline naphthalene-1,3-disulfonate & 13-NDSA & & LC-MS/MS & [S6] & 0.02 & $\mathrm{M}, \mathrm{W}$ \\
\hline
\end{tabular}




\begin{tabular}{|c|c|c|c|c|c|c|}
\hline tris-2-chloropropyl phosphate & TCPP & $13674-84-5$ & GC-MS & {$[\mathrm{S} 7]$} & 0.05 & W \\
\hline tris-2-chloroethyl phosphate & TCEP & $115-96-8$ & GC-MS & {$[\mathrm{S} 7]$} & 0.05 & W \\
\hline N,N-Diethyl-m-toluamid & DEET & & GC-MS & {$[\mathrm{S} 8]$} & 0.05 & $\mathrm{M}, \mathrm{W}$ \\
\hline Bayrepel-acid $^{3)}$ & BayAcid & & GC-MS & {$[\mathrm{S} 8]$} & 0.05 & W \\
\hline Diclofenac & DICLO & $1530-7-86-5$ & GC-MS & [S9] & 0.05 & W \\
\hline Carbamazepine & CARBA & $298-46-4$ & GC-MS & [S9] & 0.05 & W \\
\hline clofibric acid & CLOFI & $882-09-7$ & GC-MS & [S9] & 0.05 & W \\
\hline ibuprofen & IBU & $1568-7-21-1$ & GC-MS & [S9] & 0.05 & W \\
\hline 2,4-Dichlorobenzoic acid & DCBA & $50-84-0$ & GC-MS & {$[\mathrm{S} 9]$} & 0.05 & W \\
\hline Mecoprop & MCPP & $93-65-2$ & GC-MS & [S9] & 0.05 & W \\
\hline Galaxolide & HHCB & $1222-05-5$ & CLSA-GC/MS & {$[\mathrm{S} 10]$} & 0.02 & $\mathrm{M}, \mathrm{W}$ \\
\hline Tonalide & AHTN & $1506-02-1$ & CLSA-GC/MS & [S10] & 0.02 & M, W \\
\hline musk xylene & MX & $81-15-2$ & CLSA-GC/MS & [S10] & 0.03 & M, W \\
\hline musk ketone & MK & $81-14-1$ & CLSA-GC/MS & [S10] & 0.03 & $\mathrm{M}, \mathrm{W}$ \\
\hline
\end{tabular}

$\left.{ }^{1}\right)$ the precursor compounds LAS were also determined.

$\left.{ }^{2}\right)$ M: monitoring study; W: WWTP study. Additionally atrazine, simazine, metamitron, terbutylazine and desethylatrazine were analyzed in the monitoring, without positive findings above LOQ $(0.05 \mathrm{Fg} / \mathrm{L})$.

${ }^{3}$ ) The precursor compound Bayrepel was also determined 
Table S2: Characteristics of the eight wastewater treatment plants investigate in the first monitoring campaign in four European countries.

\begin{tabular}{|l|l|l|l|}
\hline & minimum & maximum & median \\
\hline dry weather flow $\left(\mathrm{m}^{3} / \mathrm{d}\right)$ & $1^{`} 100$ & $240^{`} 000$ & $45^{`} 000$ \\
\hline population equivalents & $3^{`} 400$ & $1^{`} 600^{`} 000$ & $270^{`} 000$ \\
\hline percentage industrial wastewater & 0 & 80 & 15 \\
\hline denitrification & no: 2 & yes: 6 & yes \\
\hline enhanced p removal & no: 3 & yes: 5 & yes \\
\hline hydraulic retention time HRT $(\mathrm{h})$ & 17 & 46 & 22 \\
\hline sludge retention time SRT $(\mathrm{d})$ & 8 & 38 & 15 \\
\hline sludge load (kg BOD/kg sludge * d) & 0.006 & 0.15 & 0.05 \\
\hline $\begin{array}{l}\text { biological oxygen demand } \\
\text { BOD }\end{array}$ out $(\mathrm{mg} / \mathrm{L})$ & 2 & 20 & 4 \\
\hline $\begin{array}{l}\text { chemical oxygen demand } \\
\text { COD }_{\text {out }}(\mathrm{mg} / \mathrm{L})\end{array}$ & 22 & 93 & 37 \\
\hline
\end{tabular}


Water Cycle Spreading Index:

Based on the equation:

$W C S I=\frac{\text { effluent concentration }}{\text { normalized removal in WWTP }}=\frac{C_{\text {in }} \cdot C_{\text {out }}}{C_{\text {in }}-C_{\text {out }}}$

two additional scenarios were calculated:

1. All compounds have the same influent concentration $(10 \mu \mathrm{g} / \mathrm{L})$, but exhibit removals between 99 and $1 \%$, resulting in effluent concentrations ranging from 0.1 to $9.9 \mu \mathrm{g} / \mathrm{L}$. This results in WCSI varying between 0.1 (for 99\% removal) and $990 \mu \mathrm{g} / \mathrm{L}$ (for $0.1 \%$ removal) (Fig. S1).

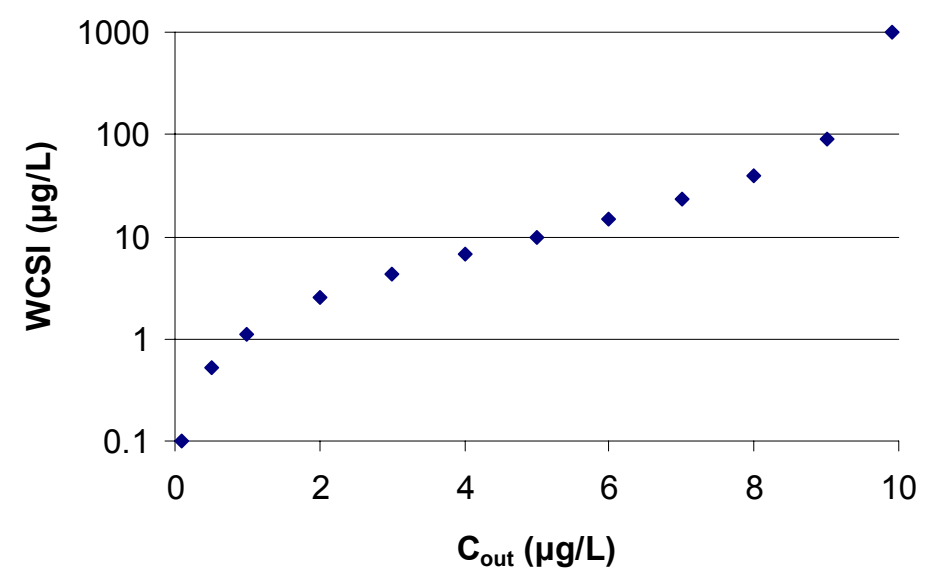

2. All compounds exhibit the same extent of removal (50\%), but vary in influent concentration between $1 \mu \mathrm{g} / 1$ and $1000 \mu \mathrm{g} / \mathrm{L}$. The effluent concentrations, thus vary between $0.5 \mu \mathrm{g} / \mathrm{L}$ and 500 $\mu \mathrm{g} / \mathrm{L}$. Then the WCSI varies between 1 and $1000 \mu \mathrm{g} / \mathrm{L}$ and is a linear function of the effluent and influent concentration, respectively (Fig. S2).

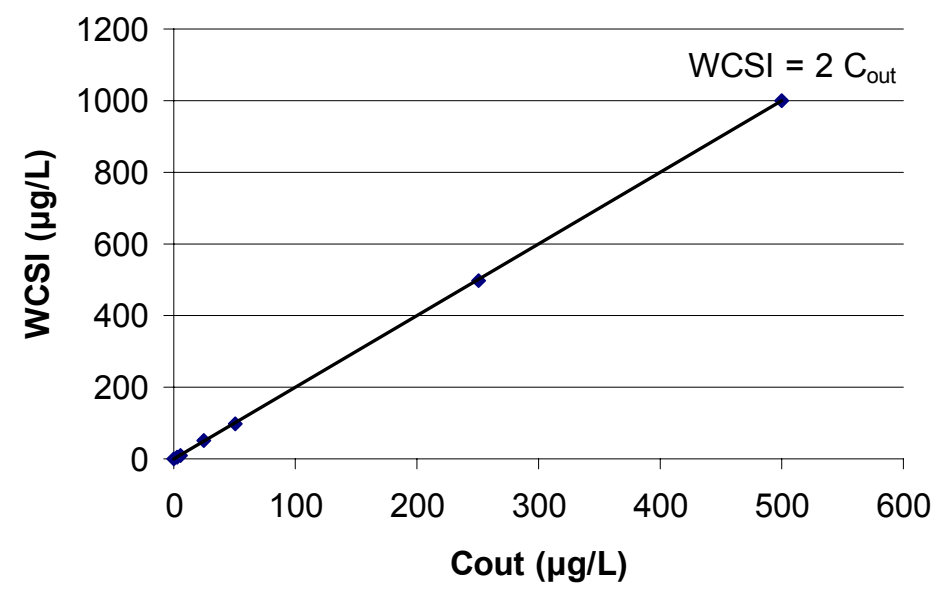




\section{References:}

(S1) Eichhorn, P.; Knepper, T.P. $\alpha, \beta$-unsaturated sulfophenylcarboxylates as degradation intermediates of linear alkylbenzene sulfonates: evidence for $\omega$-oxygenation followed by $\beta$ oxidations by liquid chromatography-mass spectrometry. Environ. Toxicol. Chem. 2002, 21, 1-8.

(S2) Knepper, T.; Werner, A.; Bogenschuetz, G. Determination of synthetic chelating agents in surface and wastewater by ion chromatography-mass spectrometry. J. Chromatogr. A 2005, 1085, 240-246.

(S3) Gonzales, S.; Petrovic, M.; Barcelo, D. Simultaneous extraction and fate of linear alkylbenzene sulfonates, coconut diethanol amides, nonlyphenol ethoxylates and their degradation products in wastewater treatment plants, receiving coastal waters and sdeiments in the Catalonia area (NE Spain). J. Chromatogr. A 2004, 1052, 111-120.

(S4) Kloepfer, A.; Jekel, M.; Reemtsma, T. Determination of benzothiazoles from complex aqueous samples by liquid chromatography-mass spectrometry following solid-phase extraction. $J$. Chromatogr. A 2004, 1058, 81-88.

(S5) Weiss, S.; Reemtsma, T. Determination of benzotriazoles corrosion inhibitors from aqueous environmental samples by liquid chromatography-electrospray ionization-tandem mass spectrometry. Anal. Chem. 2005, 77, 7415-7420.

(S6) Storm, T.; Reemtsma, T.; Jekel, M. Use of volatile amines as ion pairing agents for the high performance liquid chromatography - mass spectrometric determination of aromatic sulfonates in industrial wastewater. J. Chromatogr. A 1999, 854, 175-185.

(S7) Meyer, J.; Bester, K. Organophosphate flame retardants and plasticisers in wastewater treatment plants. J. Environ. Monit. 2004, 6, 599-605.

(S8) Knepper, T.P. Analysis and mass spectrometric characterization of the insect repellent Bayrepel and its main metabolite Bayrepel-acid. J. Chromatogr. A 2004, 1046, 159-166.

(S9) Bernhard, M.; Mueller, J.; Knepper, T.P. Biodegradation of persistent polar pollutants in wastewater: comparison of an optimized lab-scale membrane bioreactor and activated sludge treatment. Water Res. 2005, subm.

(S10) Mitjans, D.; Ventura, F. Determination of musks and other fragrance compounds at $\mathrm{ng} / \mathrm{L}$ levels using CLSA (closed loop stripping analysis) and GC/MS detection. Water Sci. Technol. 2004, 50, 119-123. 